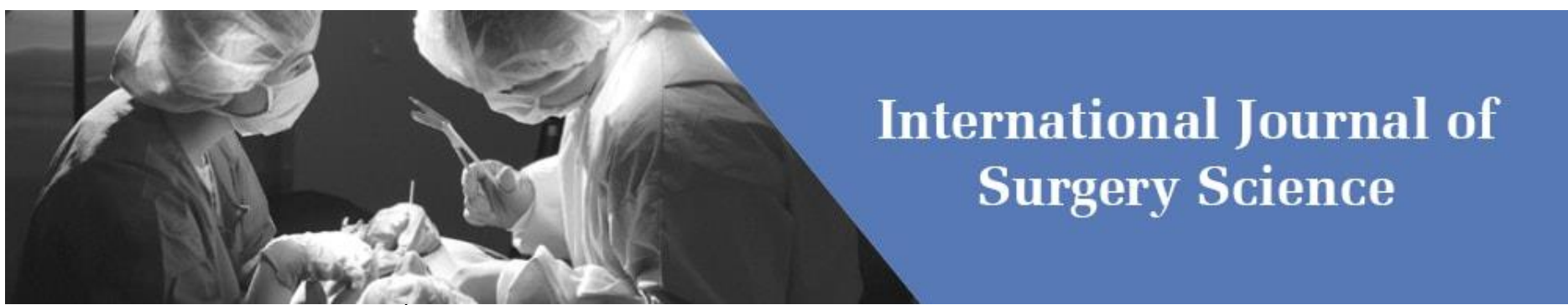

E-ISSN: 2616-3470

P-ISSN: 2616-3462

(c) Surgery Science

2019; 3(2): 145-147

Received: 01-05-2019

Accepted: 30-05-2019

Rajesh Kumar Rathore

Associate Professor, Department of

General Surgery, Government

Medical College, Dungarpur,

Rajasthan, India

\section{Comparative study of management of second and third degree Hemorrhoids with injection Sclerotherapy using Polidocanol}

\author{
Rajesh Kumar Rathore
}

DOI: https://doi.org/10.33545/surgery.2019.v3.i2c.30

\section{Abstract}

Background: Hemorrhoid is the anal disease and causes major morbidity and economic burden. Surgical treatment causes major complications. Injection Sclerotherapy is widely used in the treatment of hemorrhoids. So the present study was carried out to find the comparative effectiveness of Injection Sclerotherapy using Polidocanol for the management of second and third degree hemorrhoids.

Methods: A total of 50 patients reported to the surgical outpatient department of Government Medical College, Dungarpur with second and third degree hemorrhoids were included in this study. The patients were divided into two groups ie. Second and third degree hemorrhoids $(n=25)$. Injection polidocanol was used for the Sclerotherapy with three doses at an interval of 3 weeks.

Results: In the present study most of the patients were in the age range of 51-60 year and male preponderance was high. In second degree hemorrhoids patients $96 \%$ displayed satisfactory relief as compared to third degree hemorrhoids patients only $72 \%$ elicited satisfactory outcome.

Conclusion: Thus injection Sclerotherapy using Polidocanol elicits good results with less complications and low cost. Further, the results have shown that this method is effective in second degree hemorrhoids as that of the third degree hemorrhoids.

Keywords: Hemorrhoids, injection Sclerotherapy, Polidocanol, second degree Hemorrhoids

\section{Introduction}

Haemorrhoids, also referred as piles are a pathological condition occurring at the anorectal region. Globally the incidence ranges from $50-80 \%$ and in India it affects around $75 \%$ of the population ${ }^{[1]}$. Haemorrhoids, generally has the peak prevalence at the age of 45 to 65 years and affects both the genders. The factors associated with the development of haemorrhoids encompass erect posture, constipation, straining during defecation, high fat diet and low fibre intake ${ }^{[2]}$. Based on the degree of prolapse, Goligher has graded the Haemorrhoids for the accurate treatment. Thus, first degree Haemorrhoids has visible vessels, second degree haemorrhoids prolapse with defecation and restore instantly, third degree lesions prolapse but requires manual replacement and fourth degree lesions prolapse out of the anal canal ${ }^{[3]}$. Based on the degree of haemorrhoids, the treatment strategies include dietary restriction, injection sclerotherapy, rubber band ligation, scalpel and laser surgery $[4,5]$. When compared to other treatment modalities, injection sclerotherapy is a widely employed outpatient clinical procedure to treat first and second-degree haemorrhoids. This technique is reliably safe, cost effective, less time consuming and success rate is high ${ }^{[6,7]}$. Various sclerosing agents like Sodium tetra decyl sulphate, polidocanol, sodium morrhuate and ethanolamine oleate have been used for clinical management of haemorrhoids ${ }^{[8]}$. Among the various sclerosing agents, polidocanol has a sclerosant as well as local anesthetic property. Due to this it elicits painless sclerotherapy, low incidence of allergy and anaphylaxis reactions ${ }^{[6]}$. In this back drop, the present study was conducted to study the comparative outcome of injection sclerotherapy with polidocanol for the management of second and third degree haemorrhoids.

\section{Materials and Methods}

The present study was carried out at Department of Surgery, Government Medical College, Dungarpur from May 2018-April 2019. A total of 50 patients reported to the surgical outpatient department of second and third degree hemorrhoids diagnosed by proctoscopy according to

\section{Rajesh Kumar Rathore}

Associate Professor, Department of

General Surgery, Government

Medical College, Dungarpur,

Rajasthan, India 
Golligher's classification and above 18 years of age of both sexes were included in the study. Patients with chronic diseases, undergone previous anorectal surgeries, fourth degree hemorrhoids, allergic to sclerosant and colorectal cancer patients were excluded from the study.

In this study, out of 50 patients 25 patients were presented with $2^{\text {nd }}$ degree hemorrhoids and the remaining 25 patients were presented with $3^{\text {rd }}$ degree hemorrhoids.

Group 1- 25 patients with $2^{\text {nd }}$ degree hemorrhoids

Group 2- 25 patients with $3^{\text {rd }}$ degree hemorrhoids

Injection polidocanol was used for the sclerotherapy of $2^{\text {nd }}$ and $3^{\text {rd }}$ degree piles. Injection sclerotherapy is performed using $3 \mathrm{ml}$ disposable syringe. After locating the haemorrhoids with help of proctoscope, the needle is introduced in to the center mass of veins, through the mucous membrane. It is important that the injection be made in to submucosa at the base of the haemorrhoidal tissue and not in to the haemorrhoids themselves; taking care not to enter the lumen of the vein or traverse to the sensitive margin of the dentate line. To ensure the needle is not in the lumen, plunger is drawn back before injecting. No antiseptic is necessary. The injection was placed in the upper part of the haemorrhoid, injecting $0.5-1 \mathrm{ml}$ at the each site to a maximum of $3 \mathrm{ml}$. Correction sitting must be verified by a trial injection of a small amount which should be seen to raise and pale the overlaying mucosa and be crossed by blood vessels, the so called striation sign. If no mucosal swelling occurs immediately with a trial injection, the needle must be withdrawn further. A vey great care was taken to prevent mislocation to avoid disastrous consequences. Treatment sessions for the same anatomic locations were carried out at intervals of 3 weeks. After the procedure, the patients were given a standardized treatment of Ofloxacin $200 \mathrm{mg}$ twice daily and Ornidazole 500 mg twice daily for five days along with the advice to increases vegetable intake in diet. All the patients were called for regular follow-up.

\section{Results}

50 patients were included in the study of which there were 30 males and 20 females. The age of patients were ranged from 3060 years. The maximum patients were in the age group between 51-60 years (50\%). The age distribution of the patients was displayed in Table 1.

Regarding clinical presentation, per rectal bleeding was present in all the cases $(100 \%)$, constipation was present in 40 cases $(80 \%)$ and pain during defecation was observed in 22 cases $(44 \%)$.

Table 1: Age distribution of the patients in the present study

\begin{tabular}{|c|c|c|}
\hline Age group (in Years) & No of Patients & Percentage (\%) \\
\hline $30-40$ & 10 & 20 \\
\hline $41-50$ & 15 & 30 \\
\hline $51-60$ & 25 & 50 \\
\hline
\end{tabular}

In the present study, patients with second degree hemorrhoids after giving 3 doses of polidocanol at the interval of three weeks 24 patients $(96 \%)$ showed significant reduction in the size of hemorrhoids and the bleeding was reduced completely. The remaining one patient was suggested for alternative treatment. Meanwhile in the case of patients with third degree hemorrhoids, 19 patients (72\%) showed significant reduction in the size of hemorrhoids and the bleeding was reduced completely. In the remaining 6 patients (28\%), 4 patients required additional 2 doses of polidocanol at interval of 3 weeks for complete relief and the other 2 patients needed other procedure (Table 2).

Table 2: Distribution of patients according to response of sclerotherapy

\begin{tabular}{|c|c|c|c|}
\hline $\begin{array}{c}\text { Types of } \\
\text { haemorrhoids }\end{array}$ & $\begin{array}{c}\text { No of } \\
\text { patients }\end{array}$ & $\begin{array}{c}\text { No of patients } \\
\text { cured }\end{array}$ & $\begin{array}{c}\text { Percentage } \\
(\mathbf{\%})\end{array}$ \\
\hline Second degree & 25 & 24 & 96 \\
\hline Third degree & 25 & 19 & 72 \\
\hline
\end{tabular}

\section{Discussion}

Hemorrhoid is a serious anal disease affecting the global population which causes significant morbidity and also imposes economic burden among the patients. Hemorrhoidectomy is the mainstay treatment, post-operative it causes serious complication like pain, long hospital stay, bleeding and anal stricture. So, minimal invasive treatment methods are required for the management of hemorrhoid without resection ${ }^{[9]}$.

Injection sclerotherapy is very effective and minimal invasive procedure for the treatment of hemorrhoids. Polidocanol is a major sclerosant used in the sclerotherapy and employed in the management of various pathological conditions varicose veins, esophageal variceal, vascular disease, hemangiomas, internal hemorrhoids and cystic diseases. In 2010, polidocanol is approved by the Food and Drug Administration for the treatment of hemorrhoids. So the present study was done to evaluate the effect of polidocanol in the management of second and third degree hemorrhoids ${ }^{[10]}$.

In the present study higher number patients aged ranged from 51-60 years are affected with hemorrhoids. In a study conducted by Rizwan Mansoor Khan et al. the prevalence of hemorrhoids are higher in patients with age more than 40 years ${ }^{[11]}$. In our study, male preponderance is higher of around $60 \%$ and it consistent with the study done by Rizwan Mansoor Khan et al. where the prevalence of male is higher $(75.9 \%)$.

Regarding clinical presentation, constipation was seen in all patients followed by per rectal bleeding in $80 \%$ of the cases. However, in a study conducted by Rohit et al. per rectal bleeding is the major clinical presentation present in all the patients as that of the constipation ${ }^{[12]}$.

In the present study, injection sclerotherapy with polidocanol showed satisfactory outcome in the management of hemorrhoids. However, polidocanol treatment showed better efficacy in second degree hemorrhoids, out of 25 patients, $96 \%$ patients were cured completely at 3 doses at the time interval for three weeks. Meanwhile in patients with third degree hemorrhoids only $72 \%$ of the patients were cured completely. Thus in this study, polidocanol treatment was effective in treatment for second degree hemorrhoids as that of the third degree hemorrhoids. Similar to our reports, study conducted by Mukhopadhyay et al. at three doses of injection $89.66 \%$, patients had satisfactory results ${ }^{[13]}$.

Thus in conclusion, polidocanol is an effective and safe sclerosant for the treatment of second and third degree haemorrhoids. Further, injection sclerotherapy is a reliable method for the non-surgical management of haemorrhoids and it is found to be safe and cost effective.

\section{References}

1. Ali SA, Shoeb MFR. Study of risk factors and clinical features of hemorrhoids. Int Surg J. 2017; 4:1936-9.

2. Halverson A, Haemorrhoids MD. Clin Dis Colon Rectal Surg. 2007; 20:17-85.

3. Sneider EB, Maykel A. Diagnosis and management of 
symptomatic haemorrhoids. Surg Clin North Am. 2010; 90: 17-32.

4. Corman ML. Haemorrhoids. In: Corman ML (ed) Colon and rectal surgery, $5^{\text {th }}$ edn. Williams\&Wilkins, 2005.

5. Sim AJW, Murie JA, Mackenzie I. Comparison of rubber band ligation and sclerosant injection for first and second degree haemorrhoids: a prospective clinical trial. Acta Chir Scand. 1981; 147:717-720.

6. Chauhan VF. Prospective comparative study of sclerotherapy by hypertonic and absolute alcohol for the treatment of haemorrhoids. Gujarat Med J. 2014; 69:82-6.

7. Ohinng GV, Machicado GA, Jensen DM. Definitive therapy for internal haemorrhoids New Opportunities and Options. Rev Gastroenterol Disord. 2009; 9:16-26.

8. Parsons ME. Sclerotherapy basics. Dermatol Clin. 2004; 22:501-8.

9. Tomiki Y, Ono S, Aoki J, Takahashi R, Sakamoto K. Endoscopic sclerotherapy with aluminum potassium sulfate and tannic acid for internal hemorrhoids. Endoscopy. 2014; 46:E114.

10. Zheng X, Wei QM, Zhang H. Novel Developments in Polidocanol Sclerotherapy: A Review. JBM. 2018; 6:31-41.

11. Rizwan MK, Malik I, Ansari AH, Zulkifle M, Ehtisham. A Study on associated risk factors of Haemorrhoids. J Biol Sci Opini. 2015; 3:36-38.

12. Rohit DK, Thakur O, Verma RS, Pandey G. Effectivity of injection sclerotherapy with sodium tetra decyl sulphate in the management of first and second-degree hemorrhoids. Int Surg J. 2018; 5:897-903.

13. Madhumita M, Avijit R, Gautam P, Abhiram M, Aveesha B, Aditya M, Manas K, Rahaman QM. Effectivity of Injection Sclerotherapy with Polidocanol in Early Haemorrhoids. J Evol Med Dent Sci. 2014; 3:6619-6622. 